\title{
GOLF SWING VARIABILITY IN ELITE FEMALE JUNIOR GOLFERS
}

\author{
Tomáš Gryc, Jitka Marenčáková, Matěj Brožka, František Zahálka
}

\author{
Sport research centre, Faculty of Physical Education and Sport, \\ Charles University, Prague, Czech Republic
}

\begin{abstract}
High golf performance depends on ability to repeat golf swing execution with all golf clubs and reach repetitive contact between the golf club and the ball. The aim of the study was to determine the variability of golf swing execution in lower and upper body parameters for two golf clubs. Eleven elite female golfers of junior age volunteered in the study and performed 10 trials with mid-iron and with driver at laboratory conditions. 3D motion capture system was used to measure the lower and upper body movement parameters and club head speed. To assess variability the coefficient of variation and biological coefficient of variation were used. Two-tailed t test was used to identify difference between midiron and driver variability for all kinematic variables. Club effect was found at top of the backswing in shoulders tilt $(p=0.02)$ and at impact in shoulders rotation $(p=0.01)$, shoulders tilt $(p=0.02)$ and club head speed $(p=0.001)$. We did not found any significant difference in variability of body movement kinematic parameters and temporal parameters between mid-iron and driver. We found high stability of performance in temporal parameters and for lower limbs kinematic parameters at three selected moments of the golf swing for both clubs. We suggest that stability of temporal parameters is the key to achieve the effective and repeatable golf swing in all golf clubs.
\end{abstract}

Keywords

Golf swing, shoulders, pelvis, club head speed, kinematics

\section{Introduction}

Golf swing is complex motion with all body segments involved and one of its goals is to generate high club head velocity to reach maximum ball flight distances. The club head velocity is the main indicator of the energy produced during the golf swing $[1,2]$. Centeredness (sweet spot) of club head and ball contact is the key to transfer club head energy to the ball and club head path to face angle is the key parameter to reach straight ball flight or to reach intended ball flight trajectory. Players target is to get both, maximum distance and accuracy, repeatable. Coaches often uses model of the golf swing by Wiren [3], which is focused on laws and principles that should lead to invariant golf swing, regardless to differences between players (age, gender, physical fitness or morphology). Stability of the club head velocity, path-to-face angle and centeredness of contact at impact (moment of contact between the club head and the ball) leads to repeatable ball flight distance and accuracy. Although there are different conditions on the course (slope of the terrain influence mutual position of the body, the golf club and the ball) that influence the golf swing kinematics when reaching intended ball flight, players first goal should to repeatable golf swing and ball flight in ideal conditions (driving range). Kinematic analyses is often used to quantify and describe body movement during the golf swing $[4,5]$. Studies dealing with golf swing kinematics observed parameters of the upper body movement (shoulders rotation, hips rotation and X-factor-angular difference between shoulders and hips at top of the backswing) and describe its influence on club head velocity [6-8]. Lower limbs kinematics knees flexionduring the golf swing is observed in describing movement patterns of healthy and amputee players during the full golf swing or putting stroke [9-11]. Driver, long irons and mid irons are used to deal with kinematics of the body movement during the golf swing $[4,12,13]$. Execution of the golf swing with driver and long irons requires higher accuracy in ball contact to achieve intended trajectory of the ball flight than with mid and short irons.

Although there are studies dealing with movement variability between skilled and unskilled golfers [14] and between skilled males and females [15], at our best knowledge there is no study dealing with differences at movement variability in different clubs. Thus, the purpose of this study was to assess movement variability with mid iron and driver in elite female golfers of junior age. 


\section{Methods}

\section{Research sample}

Eleven right-handed elite junior female golfers volunteered in this study (age $=16.1 \pm 1.2$ years; height $=$ $1.72 \pm 0.11 \mathrm{~m}$; mass $=61.1 \pm 8.4 \mathrm{~kg} ; \mathrm{hcp}=0.9 \pm 1.8)$. Participants did not report any health problems that could affect golf swing performance for last six month. The research was approved by the local ethics committee of the Faculty of Physical Education and Sport, Charles University under Reg. No. 191/2016 and tested players and their legal representatives were acquainted with the testing process before signing their informed consent.

\section{Kinematic data}

3D kinematic analyzer CODA Motion System with and software ODIN (Charmwood Dynamics Limited, Leicestershire, England) were used to capture and operate motion data. Active markers and clusters (consisted from 4 active markers) position was captured by four CX1 sample units, automatically received by CODAHUB and managed in ODIN software. In current study CX1 sample units were placed around the player to ensure visibility of each active marker and at least three of four active markers on each cluster during the golf swing. Active markers and clusters were placed on the player's body in accordance to testing protocol prepared for golf swing assessment. Protocol was prepared in ODIN software by modifying existing protocol for gait analyses, where eight new positions for active markers were added. Gait protocol allows building a model of lower body adapted from previous studies [16-18] where in current study active markers, clusters and virtual points were used. To calculate virtual points position the pointer equipment was used. Active markers were placed on anterior superior iliac spine and posterior superior iliac spine for both sides (pelvis model and hip joint position) and dorsal aspect of fifth metatarsal head and upper ridge of the calcaneus posterior surface (foot model), Clusters were placed on thigh and shank on both sides and according to clusters position, virtual points position were calculated to identify position of the knee (medial femoral epicondyle and lateral femoral epicondyle) and ankle (medial malleolus and lateral malleolus) for both sides. Upper limbs and shoulders position was identified by active markers placed on left and right acromion (shoulders), left and right most caudal point on lateral epicondyle (elbow) and left and right most caudal-medial point on the ulnar styloid (wrist). Golf club position was identified by two active markers placed on the shaft, specifically near the grip and $0.1 \mathrm{~m}$ from the leading edge of club head.

\section{Test protocol}

Testing in laboratory conditions was carried out in the end of tournament season with two clubs (7 iron and Driver). All players were instructed to warm up individually and make few practice swings with 7 iron and driver to get familiar with conditions in the laboratory. When active markers and clusters were placed on the individual's body, players make 5 practice swings with each club to find out if all markers and clusters stay on its position. Artificial turf grass was used to launch the ball with 7 iron and to play with driver players were instructed to choose the size of the tee which matches its individual preferences. Balls were launched to the net placed 4 meters in the shot direction and the aim point for the shot was clearly marked on the wall behind the net. The tee, marked place on the turf (for 7 iron) and the aim point formed the target line which was parallel to $\mathrm{X}$-axis in coordinate system. Players used own golf clubs and were instructed to approach each stroke as they would do on the tournament. Participants were instructed to take individual period of time for physical recovery between shots.

\section{Data analysis}

Movement parameters were assessed in three key moments of the golf swing which were defined by the club head speed in x-axis (parallel to the target line) during the swing: initial position, top of the backswing and impact (Figure 1). Initial position was determinate as moment when the club head speed reached $-0.2 \mathrm{~m} \cdot \mathrm{s}^{-1}$ during backswing. Top of the backswing was determinate as the moment when the club head reached the speed of 0 during transition (when the golf club changes movement from the backswing phase to the downswing phase) and the impact moment (the moment of contact between the club face and the ball) was determinate as the moment when the club head slows down when contact with the ball. Four temporal parameters were used to assess the golf swing: backswing time, downswing time, tempo and rhythm. Backswing time was described as time between initial position and top of the backswing, downswing time as time between top of the backswing and impact, tempo as overall duration of the swing from initial position to impact and rhythm as ratio between backswing time and downswing time. Movement parameters assessed were: shoulders rotation (SR), pelvis rotation (PR) and X-factor (XF-angular difference between shoulders and hips at top of the backswing) in horizontal plane ( $\mathrm{x}-\mathrm{y}$ plane), shoulders tilt (ST) in frontal plane ( $\mathrm{x}-\mathrm{z}$ plane), right (RKF) and left (LKF) knee flexion and left arm-club shaft angle (LAG) angle in 3D angle and club head speed (CHS) at x-axis at impact. Shoulders and pelvis rotation at the top of the backswing is calculated as difference between its value at initial position and value at the top of the backswing 
to describe whole rotational movement of the selected segments. Movement variability of kinematic parameters could be quantified by coefficient of variation (CV\%) and biological coefficient of variation (BCV\%) $[19,20]$.

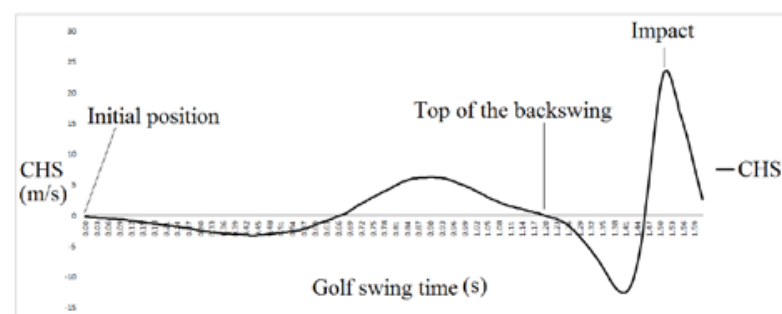

CHS-club head speed; $\mathrm{m} / \mathrm{s}$-meters per second; $s-$ second

Fig. 1: Club head speed profile in x-axis during the golf swing.

\section{Statistical analysis}

Means (X) and standard deviations (SD), standard error of the mean $\left(\mathrm{SEM} \%=\left[\left(\mathrm{SD} / \sqrt{ }_{\mathrm{n}}\right) / \mathrm{X}\right] \times 100\right.$, where $\mathrm{n}$ is number of samples and $X$ is mean), coefficient of variation $(\mathrm{CV} \%=\mathrm{SD} / \mathrm{X} \times 100$, where $\mathrm{X}$ is mean $)$ and biological coefficient of variation $(\mathrm{BCV} \%=\mathrm{CV} \%$ SEM\%) were calculated to assess the variability in all kinematic parameters. Two-tailed t-test was used in each kinematic parameter to compare mid-iron and driver at significance level of $\mathrm{p}<0.05$.

\section{Results}

All parameters in the tables are presented as absolute values. We found low variability in temporal parameters in both golf clubs (Table 1). No significant differences in kinematic parameters were found at intitial position (Table 2). In kinematic parameters (in both mid-iron and driver) we found low variability of performance in kneess flexion for both sides in each of the golf swing moment (Tables 2, 3 and 4), in X-Factor and shoulders rotation parameters at the top of the backswing (Table 3) and in club head speed parameter at Impact (Table 4). Significant differences between clubs were found in shoulders tilt parameter ( $p=0.02)$ in the top of the backswing and in shoulders rotation $(p=0.01)$, shoulders tilt $(p=0.02)$ and club head speed $(p=0.001)$ at Impact.

Table 1: Descriptive statistics for temporal parameters.

\begin{tabular}{lcccc}
\hline & \multicolumn{4}{c}{ Mid-iron (7 iron) } \\
\cline { 2 - 5 } BS (s) & MEAN & SD & CV(\%) & BCV(\%) \\
DS (s) & 1.14 & 0.04 & 3.21 & 2.19 \\
T (s) & 0.32 & 0.01 & 2.46 & 1.68 \\
R (s) & 1.46 & 0.04 & 2.78 & 1.90 \\
\hline
\end{tabular}

\begin{tabular}{lcccc}
\hline & \multicolumn{4}{c}{ Driver } \\
\cline { 2 - 5 } & MEAN & SD & CV(\%) & BCV(\%) \\
BS (s) & 1.17 & 0.04 & 3.18 & 2.15 \\
DS (s) & 0.32 & 0.01 & 1.87 & 1.27 \\
T (s) & 1.49 & 0.04 & 2.47 & 1.67 \\
R (s) & 3.67 & 0.12 & 3.35 & 2.27 \\
\hline
\end{tabular}

BS-backswing; DS-downswing; T-tempo; Rrhythm; SD—standard deviation; CV\%—coefficient of variation; BCV\%-biological coefficient of variation

Table 2: Initial position-descriptive statistics for selected kinematic parameters.

\begin{tabular}{|c|c|c|c|c|}
\hline & \multicolumn{4}{|c|}{ Mid-iron (7 iron) } \\
\hline & MEAN & SD & $\mathrm{CV}(\%)$ & $\mathrm{BCV}(\%)$ \\
\hline $\operatorname{SR}\left({ }^{\circ}\right)$ & 3.6 & 2.3 & 64.1 & 57.9 \\
\hline ST $\left({ }^{\circ}\right)$ & 9.9 & 2.3 & 23.4 & 21.2 \\
\hline $\operatorname{PR}\left({ }^{\circ}\right)$ & 5.7 & 3.3 & 58.5 & 52.8 \\
\hline$X F\left({ }^{\circ}\right)$ & 3.8 & 2.7 & 73.2 & 66.1 \\
\hline $\operatorname{LAG}\left({ }^{\circ}\right)$ & 127.7 & 23.7 & 18.5 & 16.8 \\
\hline $\operatorname{LKF}\left({ }^{\circ}\right)$ & 134.2 & 7.6 & 5.6 & 5.1 \\
\hline \multirow[t]{3}{*}{$\operatorname{RKF}\left({ }^{\circ}\right)$} & 134.4 & 3.4 & 2.5 & 2.3 \\
\hline & \multicolumn{4}{|c|}{ Driver } \\
\hline & MEAN & SD & $\mathrm{CV}(\%)$ & $\mathrm{BCV}(\%)$ \\
\hline $\operatorname{SR}\left({ }^{\circ}\right)$ & 4.5 & 2.7 & 58.9 & 53.2 \\
\hline $\operatorname{ST}\left({ }^{\circ}\right)$ & 10.9 & 2.4 & 21.8 & 19.7 \\
\hline $\operatorname{PR}\left({ }^{\circ}\right)$ & 4.4 & 2.7 & 62.3 & 56.3 \\
\hline$X F\left({ }^{\circ}\right)$ & 3.3 & 2.5 & 75.5 & 68.2 \\
\hline $\operatorname{LAG}\left({ }^{\circ}\right)$ & 125.5 & 19.3 & 15.4 & 13.9 \\
\hline $\operatorname{LKF}\left({ }^{\circ}\right)$ & 135.8 & 9.8 & 7.2 & 6.6 \\
\hline $\operatorname{RLF}\left({ }^{\circ}\right)$ & 129.9 & 8.2 & 6.3 & 5.7 \\
\hline
\end{tabular}

SR-shoulders rotation; ST-shoulders tilt; PR-pelvis rotation; XF-X-factor; LAG-left arm-club shaft; LKF-left knee flexion; RKF-right knee flexion; $\mathrm{CV} \%$ - coefficient of variation; BCV\%-biological coefficient of variation

Table 3: Top of the backswing-descriptive statistics for selected kinematic parameters.

\begin{tabular}{|c|c|c|c|c|}
\hline & \multicolumn{4}{|c|}{ Mid-iron (7 iron) } \\
\hline & MEAN & SD & $\mathrm{CV}(\%)$ & $\mathrm{BCV}(\%)$ \\
\hline $\operatorname{SR}\left({ }^{\circ}\right)$ & 110.7 & 11.3 & 10.3 & 9.3 \\
\hline ST $\left(^{\circ}\right)$ & 119.0 & 18.1 & 15.2 & 13.7 \\
\hline $\operatorname{PR}\left({ }^{\circ}\right)$ & 46.6 & 11.1 & 23.9 & 21.6 \\
\hline$X F\left({ }^{\circ}\right)$ & 65.2 & 6.4 & 9.8 & 8.9 \\
\hline $\operatorname{LAG}\left({ }^{\circ}\right)$ & 72.8 & 16.1 & 22.2 & 20.0 \\
\hline $\operatorname{LKF}\left({ }^{\circ}\right)$ & 128.2 & 7.3 & 5.7 & 5.1 \\
\hline \multirow[t]{3}{*}{$\operatorname{RKF}\left({ }^{\circ}\right)$} & 135.4 & 4.1 & 3.0 & 2.7 \\
\hline & \multicolumn{4}{|c|}{ Driver } \\
\hline & MEAN & SD & $\mathrm{CV}(\%)$ & $\mathrm{BCV}(\%)$ \\
\hline $\operatorname{SR}\left({ }^{\circ}\right)$ & 115.4 & 12.5 & 10.9 & 9.8 \\
\hline ST $\left({ }^{\circ}\right)$ & 135.9* & 21.8 & 16.1 & 14.5 \\
\hline $\operatorname{PR}\left({ }^{\circ}\right)$ & 48.4 & 12.7 & 26.3 & 23.7 \\
\hline$X F\left({ }^{\circ}\right)$ & 67.6 & 6.6 & 9.7 & 8.8 \\
\hline $\operatorname{LAG}\left({ }^{\circ}\right)$ & 72.7 & 15.1 & 20.7 & 18.7 \\
\hline $\operatorname{LKF}\left({ }^{\circ}\right)$ & 130.4 & 9.3 & 7.1 & 6.4 \\
\hline $\operatorname{RKF}\left({ }^{\circ}\right)$ & 131.0 & 6.7 & 5.1 & 4.6 \\
\hline
\end{tabular}


SR—shoulders rotation; ST—shoulders tilt; PR—pelvis rotation; XF-X-factor; LAG-left arm-club shaft; LKF-left knee flexion; RKF-right knee flexion; $\mathrm{CV} \%$ - coefficient of variation; BCV\%-biological coefficient of variation; *Significant $(p<0.05)$ between-club difference (bolded)

Table 4: Impact-descriptive statistics for selected kinematic parameters.

\begin{tabular}{|c|c|c|c|c|}
\hline & \multicolumn{4}{|c|}{ Mid-iron (7 iron) } \\
\hline & MEAN & SD & CV\% & BCV\% \\
\hline $\operatorname{SR}\left({ }^{\circ}\right)$ & 8.0 & 5.5 & 68.2 & 61.6 \\
\hline ST $\left(^{\circ}\right)$ & 19.9 & 5.6 & 27.9 & 25.3 \\
\hline $\operatorname{PR}\left({ }^{\circ}\right)$ & 50.2 & 9.2 & 18.4 & 16.6 \\
\hline$X F\left(^{\circ}\right)$ & 42.6 & 8.1 & 19.0 & 17.1 \\
\hline $\operatorname{LAG}\left({ }^{\circ}\right)$ & 143.0 & 35.0 & 24.4 & 22.1 \\
\hline $\operatorname{LKF}\left({ }^{\circ}\right)$ & 138.7 & 7.8 & 5.6 & 5.1 \\
\hline $\operatorname{RKF}\left({ }^{\circ}\right)$ & 134.7 & 5.4 & 4.0 & 3.6 \\
\hline \multirow[t]{3}{*}{$\begin{array}{l}\text { CHS } \\
\left(\mathrm{m} \cdot \mathrm{s}^{-1}\right)\end{array}$} & 20.6 & 1.8 & 8.9 & 8.1 \\
\hline & \multicolumn{4}{|c|}{ Driver } \\
\hline & MEAN & SD & CV\% & BCV\% \\
\hline $\operatorname{SR}\left({ }^{\circ}\right)$ & $14.5^{*}$ & 6.8 & 46.9 & 42.4 \\
\hline $\mathrm{ST}\left({ }^{\circ}\right)$ & $25.8^{*}$ & 6.1 & 23.5 & 21.3 \\
\hline $\operatorname{PR}\left({ }^{\circ}\right)$ & 55.4 & 9.6 & 17.3 & 15.7 \\
\hline$X F\left(^{\circ}\right)$ & 41.3 & 8.3 & 20.1 & 18.2 \\
\hline $\operatorname{LAG}\left({ }^{\circ}\right)$ & 142.6 & 29.8 & 20.9 & 18.9 \\
\hline $\operatorname{LKF}\left({ }^{\circ}\right)$ & 140.7 & 11.1 & 7.9 & 7.2 \\
\hline $\operatorname{RKF}\left({ }^{\circ}\right)$ & 130.1 & 11.1 & 8.5 & 7.7 \\
\hline $\begin{array}{l}\mathrm{CHS} \\
\left(\mathrm{m} \cdot \mathrm{s}^{-1}\right)\end{array}$ & $23.1 * *$ & 1.8 & 7.8 & 7.0 \\
\hline
\end{tabular}

SR-shoulders rotation; ST-shoulders tilt; PR-pelvis rotation; XF-X-factor; LAG-left arm-club shaft; LKF_left kneeflexion; RKF_right kneeflexion; CV\%-coefficient of variation; BCV\%-biological coefficient of variation; CHS-club head speed; *Significant $\quad(p<0.05)$ between-club difference (bolded); **Significant $\quad(\mathrm{p}<0.01)$ between-club difference (bolded)

\section{Discussion}

Significant difference between clubs in club head speed parameter at impact ( $\mathrm{p}<0.01$ ) was expected. Also significantly higher value of shoulders rotation and tilt was expected $(p<0.05)$. We suggest it is because of the ball placement at initial position for driver is recommended to be in front of left heel (front foot at stroke direction) whereas for mid-iron the ball placement is recommended to be at the middle of the stance [3]. Therefore shoulders as leading part of the body during the downswing rotate further in target direction before the golf club contact the ball. We did not found any significant differences between clubs in variability (CV\% and BCV\%) of body movement kinematic pa- rameters. Golf coaching literature and coaches opinion is that to achieve low variability in the golf swing it is important to reach high stability of movement in some key moments of the golf swing such as initial position and top of the backswing could be. In our study high stability of performance in temporal parameters were found for both clubs in the study group. Similar results in variability of timing parameters (backswing time: $\mathrm{CV} \%=3.21, \mathrm{BCV} \%=2.19$; downswing time: $\mathrm{CV} \%=$ 2.46, BCV\% = 1.68; total swing time-tempo: $\mathrm{CV} \%=$ $2.78, \mathrm{BCV} \%=1.90$ ) were presented by Keogh et al. [14] for study group consisted of adult male low handicap (hcp $=0.3 \pm 0.5$ ) golfers (backswing: $\mathrm{CV} \%=$ 3.4, $\mathrm{BCV} \%=2.3$; downswing: $\mathrm{CV} \%=5.8, \mathrm{BCV} \%=$ 2.3; total swing time: $\mathrm{CV} \%=2.3, \mathrm{BCV} \%=1.6$ ), but in their study the mean values of temporal parameters (backswing time: $0.80 \pm 0.12 \mathrm{~s}$; downswing time: $0.29 \pm 0.03 \mathrm{~s}$; total swing time: $1.07 \pm 0.14 \mathrm{~s}$ ) differs in comparison to our study results for both mid-iron (backswing time: $1.14 \pm 0.04 \mathrm{~s}$; downswing time: $0.32 \pm 0.01 \mathrm{~s}$; total swing time: $1.46 \pm 0.04 \mathrm{~s}$ ) and driver (backswing time: $1.17 \pm 0.04 \mathrm{~s}$; downswing time: $0.32 \pm 0.01 \mathrm{~s}$; total swing time: $1.49 \pm 0.04 \mathrm{~s}$ ). We found relatively large range of motion in shoulders rotation parameter $\left(110.7^{\circ} \pm 11.3^{\circ}\right)$ in the study group but stability of movement was not that high $(\mathrm{CV} \%=10.3$, $\mathrm{BCV} \%=9.3)$ as in study of Keogh et al. [14] who reports range of motion in trunk rotation of $99.3^{\circ} \pm 10.8^{\circ}$ in skill male golfers and high stability of performance also $(\mathrm{CV} \%=2.3, \mathrm{BCV} \%=1.5)$. We suggest that cause of higher body movement variability in our study group could be range of age of participants and their adolescent age which is associated with hormonal changes during maturation and age related increasing of strength in girls [21] which both effect the golf swing technique and its variability. It is well known, that there are kinematic differences in upper body movement parameters during the golf swing between genders and that females use higher range of motion in upper body segments $[7,15]$. Zheng et al. [7] reported pelvis rotation for professional female golfers $49.0^{\circ} \pm 8.0^{\circ}$ similar results we found in our study in pelvis rotation for mid-iron $46.6^{\circ} \pm 11.1^{\circ}$ and also for driver $48.4^{\circ} \pm 12.7^{\circ}$. In our study group other upper body kinematic parameters did not show such performance stability as shoulders rotation and $\mathrm{X}$-factor at top of the backswing and we suggest it is because of age range in the study group and intraindividual differences in determinants effecting movement performance during the golf swing such as flexibility, muscular strength, body mass and physical fitness. In our study we found high stability of performance for both knees at each of selected golf swing moments and for both clubs. We suggest that there is connection to high stability of movement for upper body movement parameters (shoulders rotation, X-factor) at the top of the backswing which is important fact to reach stable club head speed at impact. There are some possible limits of the study such as large age range of the study group. For future studies we recommend to take 
under account distribution of study subjects to groups based on biological age and applied full body golf swing model including head and both upper limbs. Future research should bring deeper analyze of body movement parameters during the golf swing that affect performance with respect to gender, age, physical fitness and morphological differences between subjects, explore the role of environment (different ball position, changing targets direction and distance, etc) in golf swing kinematics and its relation to "on course" performance (score, game statistics).

\section{Conclusion}

In current study significantly higher values of club head speed, shoulders rotation and shoulders tilt were found for driver when compared to mid-iron. We found high stability of performance in temporal parameters and conclude that it is important to practice rhythm and tempo with respect to individual differences between players. Also we found high stability of performance for knees flexion on both sides in each of the selected golf swing moments and for both clubs. We suggest that decreased variability in knees motion leads to correct and invariable shoulders rotation at top of the backswing and to high stability of club head speed at impact. In current study the effect of club on variability of upper and lower body movement wasn't found.

\section{Acknowledgement}

The work has been supported by research grant UNCE/HUM/032.

\section{References}

[1] Meister DW, Ladd AL, Butler EE, Zhao B, Rogers AP, Ray CJ, et al. Rotational Biomechanics of the Elite Golf Swing: Benchmarks for Amateurs. Journal of Applied Biomechanics. 2011;27:242-51. DOI: 10.1123/jab.27.3.242

[2] Meister D, Schroeder J, Butler E, Twist K, Ladd A, Rose J. Kinematic and Kinetic Analysis of the elite golf swing. 24 th Annual International Symposium On Biomechanics In Sports. 2006:TUA3-2.

[3] Wiren G. Teaching manual. Palm Beach Gardens: Greenstone Roberts Advertising; 1990.

[4] Gryc T, Zahálka F, Malý T, Malá L, Hráský P. Movement’s analysis and weight transfer during the golf swing. Journal of Physical Education and Sport. 2015;15(4):781-7. DOI: $10.7752 /$ jpes.2015.04119

[5] Erget CI, Vincent O, Weber J, Dujardin FH, Chollet D. Analysis of 3D Kinematics Concerning Three Different Clubs in Golf Swing. International Journal of Sports Medicine. 2003;24(6): 465-9. DOI: $\underline{10.1055 / \mathrm{s}-2003-41175}$

[6] Myers J, Lephart S, Tsai Y, Sell T, Smoliga J, Jolly J. The role of upper torso and pelvis rotation in driving performance during the golf swing. Journal of Sports Sciences. 2008;26(2):181-8. DOI: $10.1080 / 02640410701373543$

[7] Zheng N, Barrentine SW, Fleisig GS, Andrews JR. Swing kinematics for male and female pro golfers. International Journal of Sports Medicine. 2008;29(12):965-70.

DOI: $10.1055 / \mathrm{s}-2008-1038732$

[8] Cochran AJ, Stobbs J, Noble D. Search for the perfect swing: The proven scientific approach to fundamentally improving your game: Triumph books Chicago, IL, USA; 2005.

[9] Stastny P, Maszczyk A, Tomankova K, Kubovy P, Richtrova M, Otahal J, et al. Kinetic and Kinematic Differences in a Golf Swing in One and Both Lower Limb Amputees. Journal of Human Kinetics. 2015;48(1):33-41. DOI: 10.1515/hukin-2015-0089

[10] Gryc T, Stastny P, Zahalka F, Smolka W, Zmijewski P, Golas A, et al. Performance and Kinematic Differences in Putting Between Healthy and Disabled Elite Golfers. Journal of Human Kinetics. 2017;60(1):233-41. DOI: 10.1515/hukin-2017-0113

[11] Gryc T, Zahálka F, Malý T, Hráský P, Malá L. Movements analysis and weight transfer during the golf swing. Journal of Physical Education and Sport. 2015;15(4):781-787. DOI: $10.7752 /$ jpes.2015.04119

[12] Healy A, Moran KA, Dickson J, Hurley C, Smeaton AF, $\mathrm{O}^{\prime}$ Connor NE, et al. Analysis of the 5 iron golf swing when hitting for maximum distance. Journal of sports Sciences. 2011;29(5):1079-88. DOI: 10.1080/02640414.2011.576693

[13] Tinmark F, Hellström J, Halvorsen K, Thorstensson A. Elite golfers kinematic sequence in full-swing and partial-swing shots. Sports Biomechanics. 2010;9(4):236-44. DOI: $10.1080 / 14763141.2010 .535842$

[14] Keogh J, Bradshaw E, Hume P, Maulder P, Marnewick M, Nortje J. Biological Movement Variability During the Golf Swing. 25 International Symposium on Biomechanics in Sports 2007.

[15] Horan SA, Evans K, Kavanagh JJ. Movement variability in the golf swing of male and female skilled golfers. Medicine and Science in Sports and Exercise. 2011;43(8):1474-83. DOI: $\underline{10.1249 / \mathrm{MSS} .0 \mathrm{~b} 013 \mathrm{e} 318210 \mathrm{fe} 03}$

[16] Bell AL, Pedersen DR, Brand RA. A comparison of the accuracy of several hip center location prediction methods. J Biomech. 1990 Jan 1;23(6):617-21. DOI: 10.1016/0021-9290(90)90054-7

[17] Leardini A, Cappozzo A, Catani F, Toksvig-Larsen S, Petitto A, Sforza V, et al. Validation of a functional method for the estimation of hip joint centre location. J Biomech. 1999;32:99103. DOI: $10.1016 / \mathrm{S} 0021-9290(98) 00148-1$

[18] Cappozzo A, Catani F, Della Croce U, Leardini A. Position and orientation in space of bones during movement: anatomical frame definition and determination. Clin Biomech. 1995;10(4): 171-8. DOI: 10.1016/0268-0033(95)91394-T

[19] Heiderscheit BC. Movement variability as a clinical measure for locomotion. Journal of Applied Biomechanics. 2000;16:419-27. DOI: $10.1123 /$ jab.16.4.419

[20] Bradshaw EJ, Maulder PS, Keogh JWL. Biological movement variability during the sprint start: Performance enhancement or hindrance? Sports Biomechanics. 2007;6(3):246-60. DOI: $\underline{10.1080 / 14763140701489660}$

[21] Ramos E, Frontera WR, Llopart A, Feliciano D. Muscle strength and hormonal levels in adolescents: Gender related differences. International Journal of Sports Medicine, 1998;19(8):526-31. DOI: $\underline{10.1055 / \mathrm{s}-2007-971955}$

Tomáš Gryc, Ph.D. Sport Research Centre Faculty of Physical Education and Sport Charles University José Martího 269/31, CZ-162 52 Prague

E-mail: gryc.tomas@ftvs.cuni.cz Phone: +420 724888115 